\title{
Structural insights on biologically relevant cationic membranes by ESR spectroscopy
}

\author{
Julio H. K. Rozenfeld ${ }^{1} \cdot$ Evandro L. Duarte $^{2} \cdot$ Tiago R. Oliveira $^{3} \cdot$ M. Teresa Lamy ${ }^{2}$ (I)
}

Received: 17 June 2017 / Accepted: 28 July 2017 /Published online: 23 August 2017

(C) International Union for Pure and Applied Biophysics (IUPAB) and Springer-Verlag GmbH Germany 2017

\begin{abstract}
Cationic bilayers have been used as models to study membrane fusion, templates for polymerization and deposition of materials, carriers of nucleic acids and hydrophobic drugs, microbicidal agents and vaccine adjuvants. The versatility of these membranes depends on their structure. Electron spin resonance (ESR) spectroscopy is a powerful technique that employs hydrophobic spin labels to probe membrane structure and packing. The focus of this review is the extensive structural characterization of cationic membranes prepared with dioctadecyldimethylammonium bromide or diC14-amidine to illustrate how ESR spectroscopy can provide important structural information on bilayer thermotropic behavior, gel and fluid phases, phase coexistence, presence of bilayer interdigitation, membrane fusion and interactions with other biologically relevant molecules.
\end{abstract}

Keywords Cationic membranes - ESR spectroscopy · Spin labels $\cdot$ Lipid bilayers

This article is part of a Special Issue on 'Latin America' edited by Pietro Ciancaglini and Rosangela Itri.

M. Teresa Lamy

mtlamy@if.usp.br; http://fig.if.usp.br/ mtlamy/

1 Departamento de Biofísica, Escola Paulista de Medicina, Universidade Federal de São Paulo, R. Botucatu 862, São Paulo, SP 04023-062, Brazil

2 Instituto de Física, Universidade de São Paulo, R. do Matão 1371, São Paulo, SP 05508-090, Brazil

3 Centro de Engenharia, Modelagem e Ciências Sociais Aplicadas, Universidade Federal do ABC, R. Arcturus (Jd Antares), São Bernardo do Campo, SP, Brazil

\section{Introduction}

Membranes formed by synthetic cationic lipids were initially used to investigate membrane fusion and protein reconstitution (Südholter et al. 1982; Engberts and Hoekstra 1995). Establishment of their safety then led to cationic membranes being then employed as carriers of proteins and nucleic acids to eukaryotic cells (Zelphati et al. 2001; ur Rehman et al. 2013). This technical development enabled the study of gene function, silencing and therapy (Caracciolo and Amenitsch 2012) and stimulated the development of dozens of lipids with different cationic headgroups and hydrophobic moieties (ur Rehman et al. 2013; Junquera and Aicart 2016; Majzoub et al. 2016). In this review we focus on membranes formed by two cationic lipids: dioctadecyldimethylammonium bromide (DODAB) and diC14-amidine (Fig. 1).

Versatile DODAB membranes have been used as nucleic acid (Silva et al. 2011) and hydrophobic drug carriers (Oliveira et al. 2011; Vieira et al. 2006), as templates for polymerization and deposition of materials (Hubert et al. 2000), as biomimetic particles (Rosa et al. 2008), as microbicidal agents (Ragioto et al. 2014) and as vaccine adjuvants (Rozenfeld et al. 2012; Aps et al. 2016). Similarly, highly fusogenic diC14-amidine membranes (Oliveira et al. 2012) have successfully delivered nucleic acids (El Ouahabi et al. 1996; Ruysschaert et al. 1994) and stimulated the formation of immune responses (Tanaka et al. 2008).

The structure of cationic membranes and their assemblies with other molecules have a profound effect on their biological activity (Bertrand and Leroux 2012), so that much effort continues to be dedicated to characterizing these structures in detail (Dan and Danino 2014). In this context, electron spin resonance (ESR) spectroscopy is a powerful technique to gain structural insights because it provides a lot of information on membrane structure, polarity, viscosity and/or packing. The 
Fig. 1 Chemical structures of the cationic lipids

[dioctadecyldimethylammonium bromide (DODAB), diC14- and diC16-amidine], phospholipids [DMPG (dimyristoyl phosphatidylglycerol), DMPC (dimyristoyl phosphatidylcholine) and DPPC (dipalmitoyl

phosphatidylcholine)], spin labels (5- and 16-PCSL, 5- and 16MESL and 5- and 16-SASL) and the molecules amphotericin B and cholesterol
Cationic Lipids

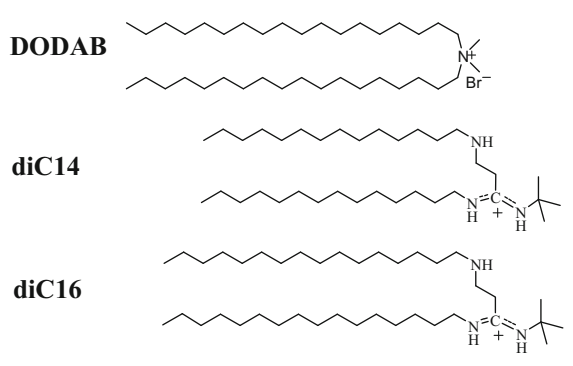

Spin Labels

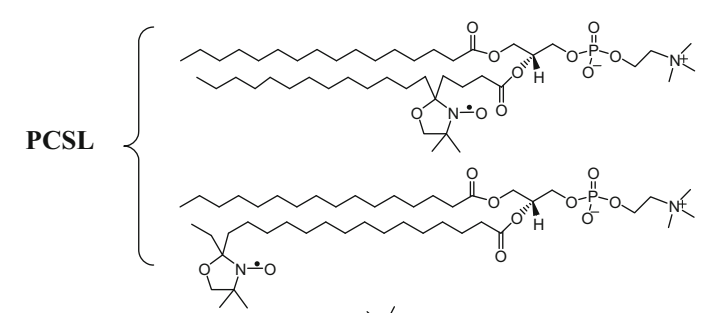

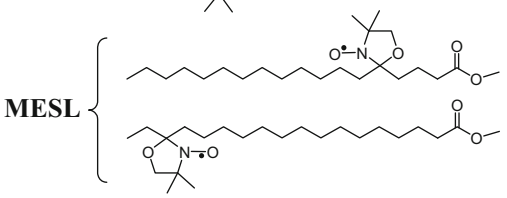

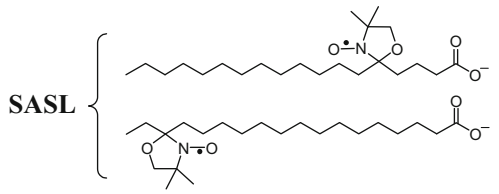

Phospholipids

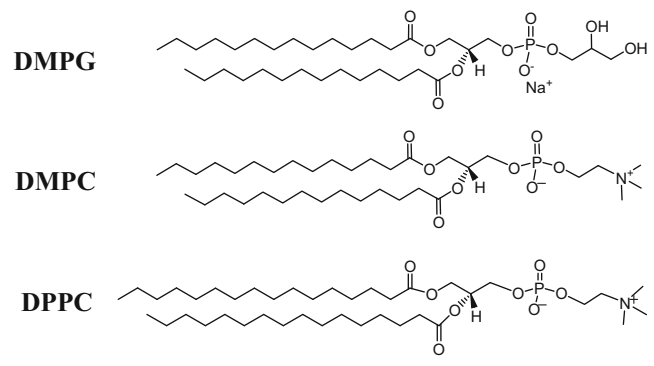

Amphotericin B

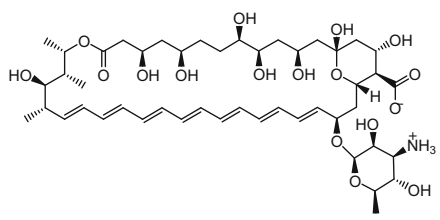

Cholesterol

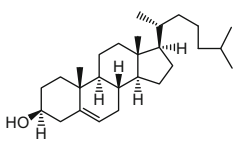

multiple applications of this technique in the structural characterization of systems containing cationic membranes are explored in this review.

\section{A short review about ESR of spin labels in lipid bilayers}

Electron spin resonance, also called electron paramagnetic resonance, is a spectroscopic technique that requires unpaired (paramagnetic) electrons. As most lipid membranes have no paramagnetic center, spin-probes are introduced into the membranes, usually in tiny amounts (less than $1 \%$ mole of probe per mole of lipid). The nitroxide radical is one of the most used spin label groups in biological systems (see, for example, Marsh 1981). A stable unpaired electron is localized in the $2 p \pi$ orbital of the nitrogen-oxygen bond, and its interaction with the nitrogen nucleus gives rise to a hyperfine structure of three lines (corresponding to $m_{1}=+1,0,-1$ ). Thus, when the nitroxide group is moving rapidly in the ESR time-scale, the signal consists of three lines of equal heights. This high degree of mobility is obtained for small spin-labeled molecules that are freely tumbling in non-viscous solutions. Increasing immobilization of the spin label leads to a differential line broadening in its ESR spectrum (see, for example, Hubbell and McConnell 1971). Hence, the ESR signal of spin labels is particularly sensitive to the environment of the probe.

Spin labels used in lipid membranes are typically spinlabeled lipids, similar to those shown in Fig. 1, derived from phospholipids, stearic acid and its methyl ester derivative and labeled at different positions along the hydrocarbon chain.

Generally, two approaches are employed to analyze the ESR signal. The first one is based on empirical parameters directly obtained from the spectrum. This method is usually not capable of separating information on order and mobility of the label, and it is not recommended if the ESR spectrum corresponds to a sum of two or more components. In this latter case, the second method, theoretical simulation of the spectrum, is an important tool to be used to obtain structural information on the two sites separately. However, the best yielded simulation of the spectrum of spin labels is strongly dependent on the initial parameters. Iterations with different initial values can lead to distinct local minima. Hence, the simulation analysis is not intended to be carried out as fully automated process, but requires the user to have an expertise on ESR theory (Schneider and Freed 1989). 


\section{Empirical parameters}

As will be discussed below, lipid membranes can display different phases depending on the temperature, $\mathrm{pH}$ and water content. Hence, the ESR technique is an important tool since the ESR spectrum of spin labels provides detailed information about the molecular mobility, order and polarity of the bilayer along the acyl chain. The maximum hyperfine splitting value, $A_{\text {max }}$, measured with 5-PCSL in a DODAB fluid bilayer (Fig. $2 \mathrm{~b}$ ) is clearly smaller than the one measured at the gel phase (Fig. 2a). Hence, $A_{\max }$ can be used to inform about the packing of the nano-environment monitored by the spin-probe. For 5PCSL in gel bilayers (Fig. 2a), it is not possible to distinguish order from mobility with empirical parameters. However, in the membrane fluid phase (Fig. 2b), due to the fast movement of 5PCSL around its long axis [in the time scale of the ESR technique, a rotational correlation time $\left.(\tau)<10^{-10} \mathrm{~s}\right)$, in addition to obtaining $A_{\text {max }}$, it is also possible to measure $A_{\text {min }}$ (Fig. 2b), and an effective order parameter, $S_{\text {eff, }}$, can be calculated as follows (Knowles et al. 1976; Gaffney 1976; Smith et al. 1976).

$S_{e f f}=\frac{A_{\|}-A_{\perp}}{A_{z z}-(1 / 2)\left(A_{x x}+A_{y y}\right)} \frac{a_{o}^{\prime}}{a_{o}}$

where $A_{\|}=A_{\max }, A_{\perp}=A_{\min }+1.4\left[1-\frac{A_{\|}-A_{\min }}{A_{z z}-(1 / 2)\left(A_{x x}+A_{y y}\right)}\right]$, and $A_{x x}, A_{y y}$ and $A_{z z}$ are the principal values of the hyperfine tensor for doxylpropane (Hubbell and McConnell 1971). The

\section{DODAB}

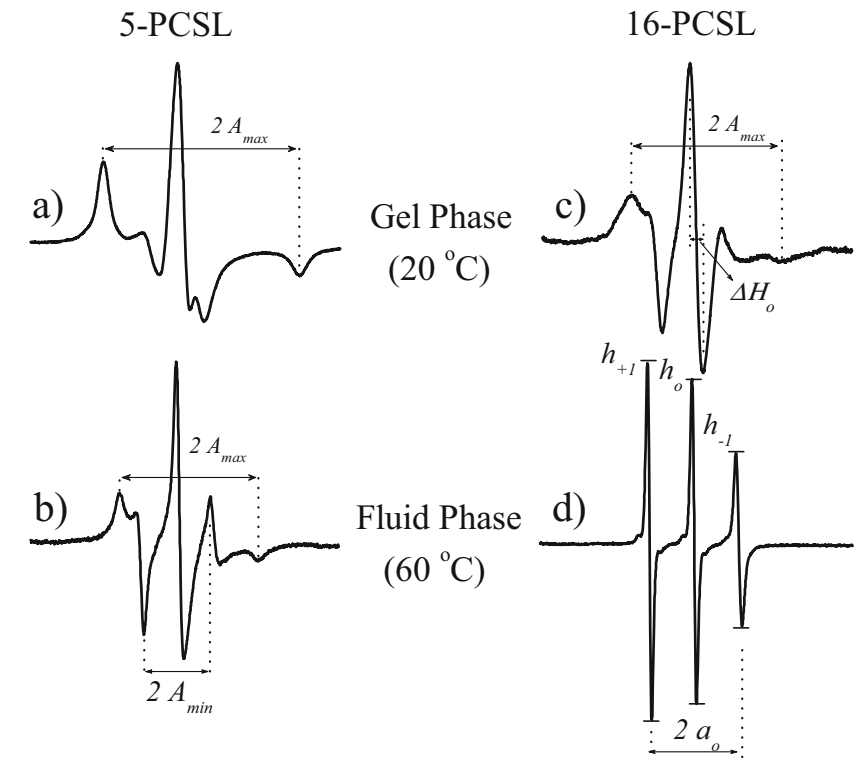

Fig. 2 a, b Electron spin resonance (ESR) spectra of 5-PCSL incorporated in $2 \mathrm{mM}$ DODAB vesicles in the gel $\left(20^{\circ} \mathrm{C}\right)(\mathbf{a})$ and fluid $\left(60^{\circ} \mathrm{C}\right)(\mathbf{b})$ phases. c, d ESR spectra of 16-PCSL incorporated in $2 \mathrm{mM}$ DODAB vesicles in the gel $\left(20^{\circ} \mathrm{C}\right)(\mathbf{c})$ and fluid $\left(60^{\circ} \mathrm{C}\right)(\mathbf{d})$ phases. Total spectra width $100 \mathrm{G}$ experimental and theoretical isotropic hyperfine splitting, $a_{0} \mathrm{e}$ $a_{0}^{\prime}$ respectively, can be calculated from the expressions:

$a_{o}=(1 / 3)\left(A_{\|}+2 A_{\perp}\right)$

$a_{o}^{\prime}=(1 / 3)\left(A_{x x}+A_{y y}+A_{z z}\right)$

Thus, when all the probe molecules are completely oriented parallel to the bilayer normal, $S_{\text {eff }}$ is equal to unity. On the other hand, $S_{\text {eff }}$ tends to zero when the spin labels rotate in a rapid isotropic motion. Hence, the effective order parameter can be understood as a measure of the orientation degree of the spin label relative to the bilayer normal. It is an "effective order parameter" as it has been shown to have some contribution from the spin label mobility (Lange et al. 1985).

Probes located at the core of the bilayer (16-PCSL in Fig. 1) have faster and more isotropic movement (Fig. 2c, d). In the bilayer fluid phase, this movement is nearly isotropic, and the amplitude of the three hyperfine lines, corresponding to $m_{1}=+$ $1,0,-1$, can be measured (Fig. 2d) $\left(10^{-11} \leq \tau \leq 3 \times 10^{-9}\right.$, corresponding to the motional narrowing regime; Hubbell and McConnell 1971). The ratios between the amplitudes of the lines, $h_{+1} / h_{o}$ and $h_{-1} / h_{o}$, get closer to unity as the probe movement becomes faster and less ordered, with the $h_{-1} / h_{o}$ ratio being more sensitive to the packing of the bilayer (see, for example, Marsh 1981). Usually, we also measure a $h_{+1} / h_{o}$ ratio in spectra like that of 16-PCSL in the gel phase (Fig. 2c), although we know it does not correspond to the ratios of the $m_{1}=+1$ and 0 hyperfine lines. However, this measurement provides good information on the bilayer structure, as it increases as the bilayer becomes less packed (see, for example, Marsh 1981). Sometimes, the packing of the bilayer can also be monitored by the width of the central field line $\left(\Delta H_{0}\right.$ shown in Fig. $2 \mathrm{c})$. It is worth noting that the chosen parameters are strongly dependent on both the spin label and the phase of the membrane.

Spin labels with the nitroxide moiety at various depths in the membrane can be used to study lipid bilayer hydration. It has been shown that, for spin labels inside a lipid bilayer, the magnitude of the nitrogen isotropic hyperfine splitting, $a_{o}$, is mainly related to the nitroxide-water hydrogen bonding (Griffith et al. 1974). Therefore, the extension of water presence into the bilayer can be estimated by the magnitude of $a_{o}$, either by directly measuring in an ESR spectrum like that of 16-PCSL in fluid DODAB (Fig. 2d) or by calculating from a spectrum like that of 5-PCSL in fluid DODAB (Fig. 2b), as mentioned above: $a_{o}=(1 / 3)\left(A_{\|}+2 A_{\perp}\right)$.

\section{ESR spectral simulation}

Some computational programs are available for theoretically simulating the ESR signal of spin labels. In this section we 
summarize the program used in the simulations shown here. This program, the Multi-Component EPR Fitting program was developed by Christian Altenbach (Althenbach 2017) and is based on the MOMD (microscopic order and macroscopic disorder) theory developed by Freed and coworkers (Schneider and Freed 1989; Budil et al. 1996).

As the parameters used have been extensively discussed in earlier publication (Schneider and Freed 1989; Budil et al. 1996), only a few of the most relevant points for performing the simulations will be mentioned here. The parameters are obtained through nonlinear least squares fitting of the experimental ESR signal based on the stochastic Liouville equation (Freed 1976). The dynamics of the spin label is characterized by $\tau$, calculated from the principal components of the axially symmetric rotational diffusion tensor, $R_{/ / /}$and $R_{\perp}$. For 5 - and 16-PCSL, for example, they represent the rotational rates of the nitroxide moiety around axes parallel and perpendicular to the hydrocarbon chain, respectively. For the simulations shown here, we found adequate evidence to assume the axially symmetric rotational diffusion tensor with $R_{/ /} / R_{\perp}=10$. The average rotational diffusion rate is defined as $\bar{R}=\sqrt[3]{R_{\perp}^{2} \cdot R_{/ /}}$ and $\tau=(6 \bar{R})^{-1}$. The local microscopic order of the spin label in the lipid bilayer is characterized by the order parameter, $S_{0}$, calculated from the best parameters obtained from the first terms of the expansion of the ordering potential in generalized spherical harmonics (Schneider and Freed 1989). From the hyperfine tensor components $A_{x x}, A_{y y}$ and $A_{z z}$, an isotropic hyperfine splitting can be calculated, $a_{o}=\left(A_{x x}+A_{y y}+A_{z z}\right) / 3$.

\section{Thermotropic behavior: monitoring gel and fluid-lipid bilayer phases by ESR}

Temperature affects the organization of lipids in a bilayer, resulting in different phases. The gel phase consists of molecules whose hydrocarbon chains assume a nearly all-trans conformation, packing in an array with strong van der Waals interactions and restricted molecular motions (Mason 1998; Evans and Wennerström 1999). Heating leads to the melting of lipid molecules to a fluid liquid-crystalline phase, in which van der Waals interactions are decreased, hydrocarbon chain conformational disorder predominates and translational and flip-flop motions occur more frequently (Mason 1998; Evans and Wennerström 1999). These reversible gel-fluid transitions alter membrane permeability and can be important for drug delivery applications (Anyarambhatla and Needham 1999; Ponce et al. 2006).

The phase transition of lipid bilayers can be tracked by qualitative changes in ESR spectra as well as by quantitative changes, such as the changes in the empirical parameters maximum hyperfine splitting $\left(A_{\max }\right)$ and/or the $h_{+1} / h_{0}$ ratio, or bilayer order parameter and rotational correlation times, calculated from spectra simulations, as discussed above. Hence, ESR spectroscopy can provide important information on the structural behavior of cationic membranes, such as the effect of $\mathrm{pH}$, ionic strength and the interaction with other molecules. Moreover, it can detect the presence of hysteresis and the coexistence of different phases.

Differently from DODAB, whose quaternary ammonium group is charged regardless of the $\mathrm{pH}$ (Fig. 1), diC14-amidine has two titratable groups, an amine and an amidine (Pector et al. 1998) (Fig. 1). Depending on the ionic strength, the amine and the amidine acid dissociation constant $(\mathrm{pKa}$ ) values vary from 4.6 and 8.8 (in $30 \mathrm{mM} \mathrm{NaCl}$ ) to 5.2 and 9.4 (in $130 \mathrm{mM} \mathrm{NaCl}$ ), respectively. Hence, at the physiological $\mathrm{pH}$ of 7.4, diC14-amidine should have one positive charge due to amidine protonation (Pector et al. 1998). The effect of $\mathrm{pH}$ on the structure of diC14-amidine bilayers was studied employing spin labels that probe the surface (5-PCSL) and the core (16-PCSL) of the bilayers (Benatti et al. 2004).

The ESR of spin labels incorporated in diC14-amidine bilayers showed that the membrane structure was extremely sensitive to the $\mathrm{pH}$ value of the medium, as expected. $A_{\max }$ values (outer hyperfine splitting) obtained from the spectra of a spin-probe close to the interface, 5-PCSL, and $h_{+1} / h_{0}$ ratios obtained from the spectra of a spin-probe at the bilayer core, 16-PCSL, show that diC14-amidine bilayers present a lower phase transition temperature at $\mathrm{pH} 3.0$ when compared to pH 7.4 (Benatti et al. 2004) (Fig. 3a, b). This finding is in agreement with the relative decrease in gel phase organization/packing at low $\mathrm{pH}$ (lower $A_{\max }$ values and higher $h_{+} / h_{0}$ ratios) at both the bilayer surface and the core, due to strong headgroup-headgroup electrostatic repulsion caused by the presence of the protonated amine and amidine groups. Interestingly, increasing the ionic strength (by adding $150 \mathrm{mM}$ $\mathrm{NaCl}$ to a $20 \mathrm{mM}$ phosphate buffer) did not significantly alter the phase transition temperature of diC14-amidine at $\mathrm{pH} 3.0$ and 7.4, monitored by a spin label close to the bilayer interface (Fig. 3a), as would be expected from the electrostatic screening of charged headgroups. However, it did alter the transition profile at $\mathrm{pH} 3.0$, monitored by a nitroxide at the bilayer core (Fig. 3b), from a smooth transition at low ionic strength to a sharp one at high ionic strength (Benatti et al. 2004).

As expected, DODAB ESR spectra are $\mathrm{pH}$ independent. Fig. $3 \mathrm{c}$ and $\mathrm{d}$ show that its gel-fluid transition is quite sharp and highly sensitive to the presence of ions in solution, increasing by about $4{ }^{\circ} \mathrm{C}$ with the addition of $150 \mathrm{mM} \mathrm{NaCl}$ to a 20 mM HEPES buffer pH 7.4 (Benatti et al. 2007). This indicates that the gel-fluid transition temperature of monocharged diC14-amidine ( $\mathrm{pH}$ 7.4) is less sensitive to the presence of salt than is mono-charged DODAB. It is interesting to note that DODAB with its small headgroup and 18-C chains displays a much sharper gel-fluid transition (Fig. 3c, d) than the diC14-amidine, even though the gel phase of the latter (at $\mathrm{pH}$ 7.4) is more packed than that of DODAD: higher $A_{\max }$ 
Fig. 3 a, b Temperature dependence of the outer hyperfine splitting $\left(A_{\max }\right)$ measured on the ESR spectra of 5-PCSL in $\mathrm{diC}_{14^{-}}$ amidine (a) and ratio of low and central field line amplitudes $\left(h_{+1}\right)$ $h_{o}$ ) measured on the ESR spectra of 16-PCSL in $\mathrm{diC}_{14}$-amidine at low (solid symbol) and high (open symbol) ionic strength and at pH 3.0 (circle) and 7.4 (triangle) (b). c, d Temperature dependence of the $A_{\max }$ measured on the ESR spectra of 5-PCSL in $\operatorname{DODAB}(\mathbf{c})$ and $h_{+1} / h_{o}$ measured on the ESR spectra of 16-PCSL in DODAB at low (solid symbol) and high (open symbol) ionic strength and at cooling (square) and heating (star) cycles (d). (Adapted from Benatti et al. 2004, copyright 2004 Elsevier; and from Benatti et al. 2007, copyright 2007 Elsevier)

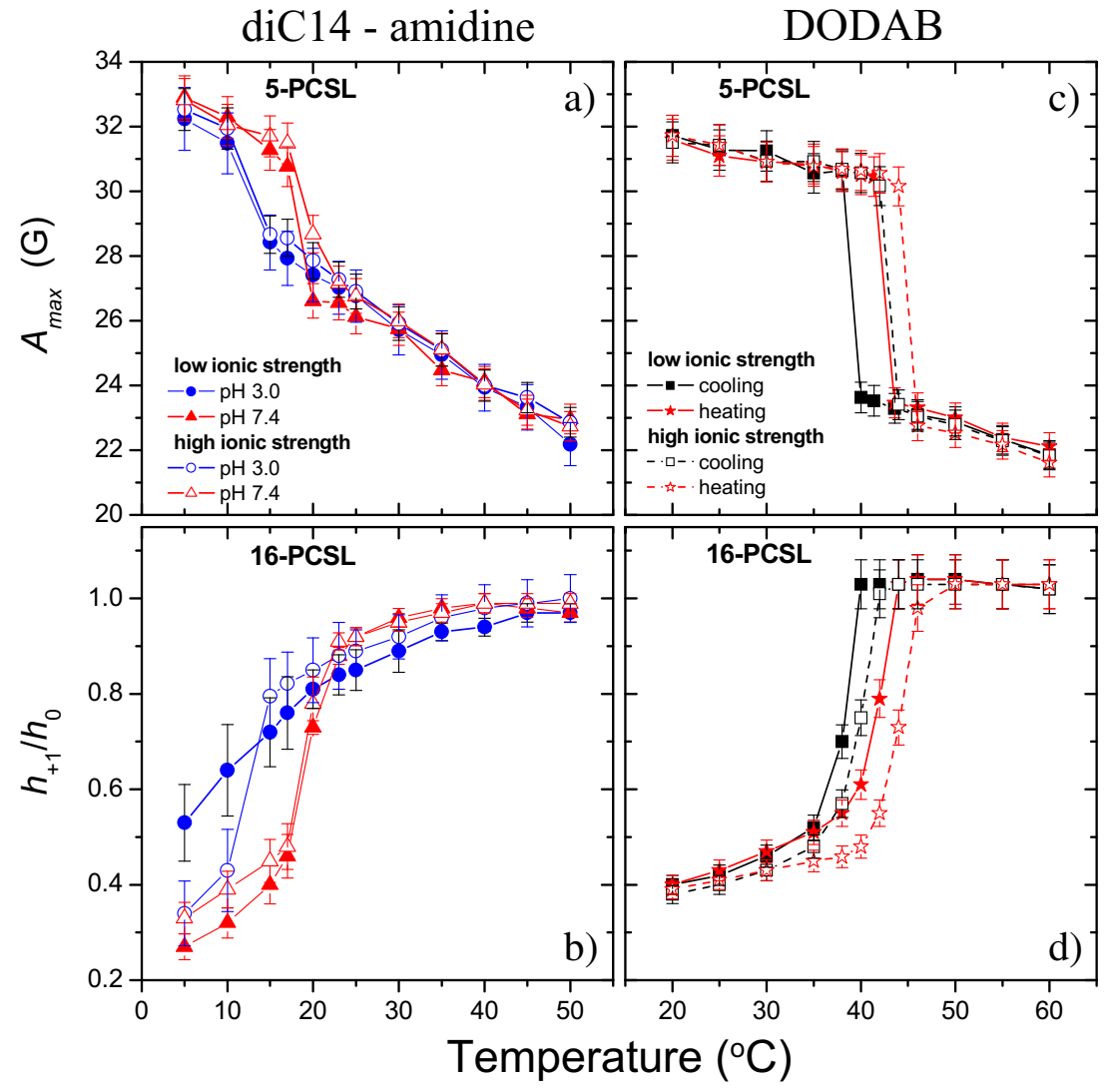

values and lower $h_{+1} / h_{0}$ ratios (Fig. 3). The structure of the gel phase of diC14-amidine will be discussed further below.

Figure $3 \mathrm{c}$ and $\mathrm{d}$ also show the significant hysteresis observed with DODAB bilayers: the transition temperature (Tm) decreases around $3{ }^{\circ} \mathrm{C}$ from heating to cooling (Benatti et al. 2007). This hysteresis was previously observed in spectra of spin labels 5- and 16-MESL and 5- and 16-SASL embedded in the cationic bilayer (Benatti et al. 2001). For example, at $40{ }^{\circ} \mathrm{C}$, the spectra of 16-MESL obtained upon heating and cooling the sample are very different, showing that the DODAB bilayer at this temperature is more fluid upon cooling than heating (Fig. 4).

Gel-fluid transitions of DODAB bilayers have been extensively investigated using differential scanning calorimetry (DSC), providing important information on transition temperature (Tm), enthalpy and cooperativity (Blandamer et al. 1992, 1996; Saveyn et al. 2007). DSC experiments also showed that the phase transition temperatures are different upon heating and cooling of the samples (Cocquyt et al. 2005; Saveyn et al. 2007). Hence, the different transition paths from one phase to the other can be also followed by ESR spectroscopy, making it a useful technique to study hysteresis in lipid bilayers. Moreover, ESR provides information on the nano-structure of the two phases.

In addition to hysteresis, the spectrum profile can also demonstrate the coexistence of the gel and fluid phases at the same temperature. If a spin label senses these two different phases, its spectrum will be a sum of the two microenvironments probed - if the exchange rate between these microenvironments is slow in the relevant time scale, set by the microwave frequency (nanoseconds). Furthermore, in order to observe

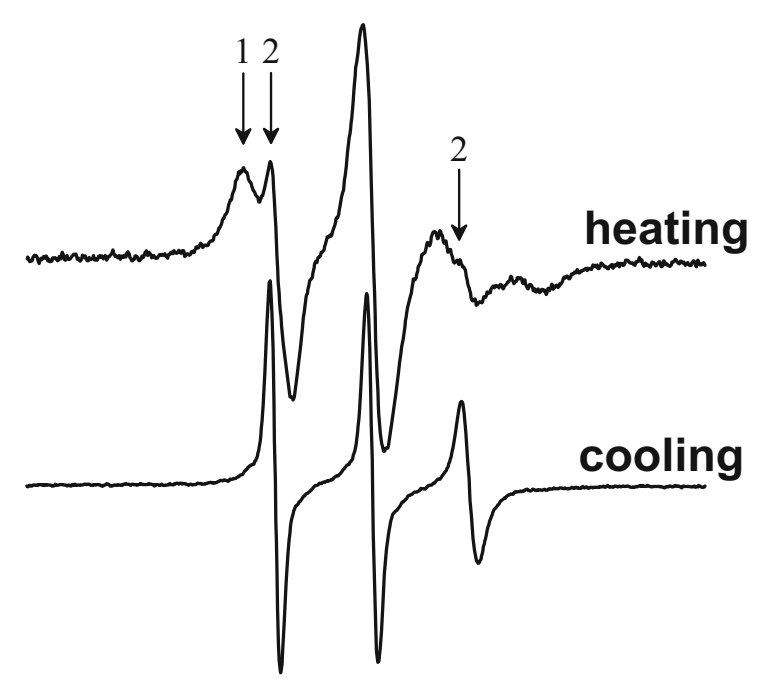

Fig. 4 The hysteresis effect in non-sonicated DODAB dispersions at $40^{\circ} \mathrm{C}$, evidenced by the different 16-MESL ESR spectra obtained upon cooling and heating the sample. Total spectra width $100 \mathrm{G}$. Arrows indicate gel (1) and fluid (2) phase features. (Adapted from Benatti et al. 2001, copyright 2001 Elsevier) 
these two microenvironments in the combined spectrum, differences in their spectral profiles are necessary. Also, a fast exchange rate between microenvironments would average out their differences (Riske et al. 2003, 2009). Spin labels located at the end of the hydrocarbon chains are better in evaluating changes along the melting process since they experience more freedom of movement when the bilayer is in the fluid phase than those located closer to the headgroups. That is the case of 16-PCSL, whose ESR spectra were the only ones that could resolve the presence of two distinct microenvironments in phase transitions of anionic dimyristoyl phosphatidylglycerol (DMPG in Fig. 1) bilayers (Riske et al. 2003, 2009).

Gel and fluid phases coexistence is present in the DODAB spectrum monitored by different probes labeled at the 16th carbon atom: 16-MESL, 16-SASL and 16-PCSL (Benatti et al. 2001, 2007; Rozenfeld et al. 2015a). In Fig. 4, a gel phase feature in the ESR spectrum of DODAD at $40{ }^{\circ} \mathrm{C}$, obtained by heating the sample, is labeled with arrow 1 and fluid phase features are labeled with arrows 2 . This phase coexistence is detected at low and high ionic strength, but for high ionic strength it is evident at higher temperatures, as expected from the stabilization of the gel phase and the increase in transition temperature due to salt screening of electrostatic interactions between headgroups (Benatti et al. 2007; see Fig. 3c, d). Gel and fluid phases coexistence was also observed around the gel-fluid phase transition temperature of diC14-amidine, between $19^{\circ} \mathrm{C}$ and $21^{\circ} \mathrm{C}$ (Oliveira et al. 2012). Below $19{ }^{\circ} \mathrm{C}$ the spectrum of 16-PCSL incorporated in diC14-amidine bilayer is typical of a membrane gel phase, and at above $22^{\circ} \mathrm{C}$, the spectra are typical of a fluid bilayer. In contrast, spectra in bilayers of the zwitterionic analog dimyristoyl phosphatidylcholine (DMPC in Fig. 1) indicate a rigid bilayer up to $23{ }^{\circ} \mathrm{C}$ and a fluid one at and above $24{ }^{\circ} \mathrm{C}$ (Oliveira et al. 2012). These ESR data are in good agreement with DSC thermograms of diC14-amidine (Fig. 5), which indicate a broad gel-fluid transition that ends before $22{ }^{\circ} \mathrm{C}$, while DMPC displays a sharp gel-fluid transition at $23.8^{\circ} \mathrm{C}$ (Oliveira et al. 2012).

It is noteworthy that while fluorescent probes (Benatti et al. 1999) and spin labels (Benatti et al. 2001) were able to track the phase transition of DODAB bilayers and yield the same transition temperatures, fluorescent probes were unable to detect phase coexistence around the transition temperature. Hence, ESR spectroscopy is generally considered to be a more sensitive technique than fluorescence spectroscopy to detect structural changes along the phase transition of the lipid bilayers.

Indeed, ESR spectroscopy also revealed the coexistence of gel and fluid domains at temperatures between the pre- and main transitions of dipalmitoyl phosphatidylcholine (DPPC in Fig. 1) and its anionic analog dipalmitoyl phosphatidylglycerol (DPPG) (Riske et al. 2009). It has also been employed to assess different membrane structural ensembles, as described in the following section.

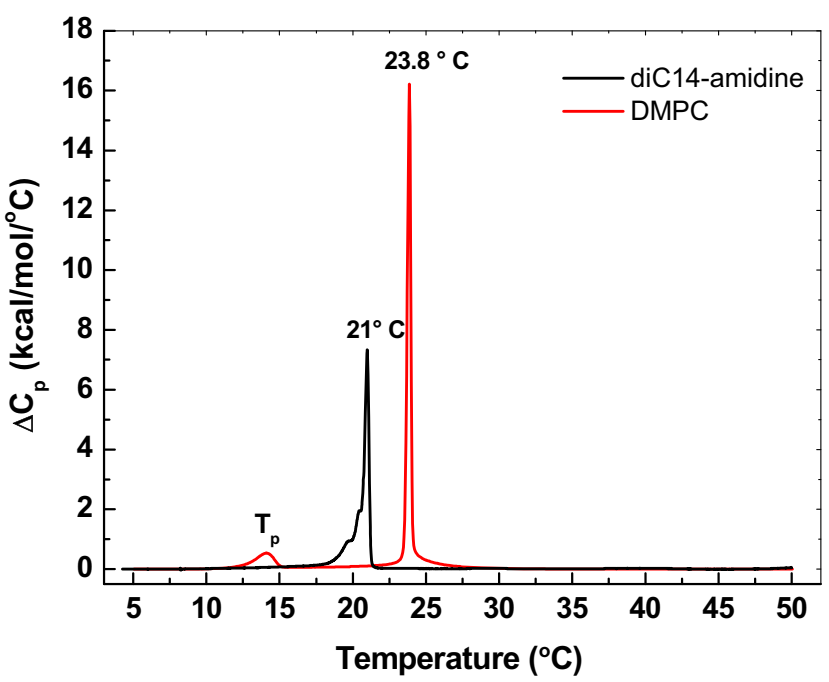

Fig. 5 Excess heat capacity $\left(\Delta C_{p}\right)$ of diC14-amidine and DMPC $(10 \mathrm{mM})$ at $\mathrm{pH}$ 7.4. Phase transition temperatures are indicated. Arrow 1 indicates gel phase feature, arrows 2 indicate fluid phase features. (Reprinted from Oliveira et al. 2012, copyright 2012 American Chemical Society)

\section{Different gel bilayer structures: interdigitation}

Electron spin resonance spectroscopy shows that both the gel and fluid phases of DODAB bilayers are more packed/ organized close to the surface than at the core, since the spectra of spin labels probing the region close to the bilayer interface (such as 5-MESL, 5-SASL or 5-PCSL; generically 5SLs) are more anisotropic than those of spin labels probing the bilayer core (such as 16-MESL, 16-SASL or 16-PCSL; generically $16-\mathrm{SLs}$ ). This is the well-known flexibility gradient toward the bilayer core described for phospholipid bilayers (Hubbell and McConnell 1971) and observed for DODAB bilayers prepared in water, buffer or high ionic strength (Benatti et al. 2001, 2007; Rozenfeld et al. 2015a).

Unlike DODAB, gel phase bilayers of diC14-amidine do not present a flexibility gradient at physiological $\mathrm{pH}$ (7.4). Phospholipids spin-labeled at different positions of the hydrocarbon chain (5-, 7-, 10-, 12- and 16-PCSL) show similar anisotropic spectra at different depths of diC14-amidine gel phase membranes (Oliveira et al. 2012). This is in contrast with the spectra observed for the same probes embedded in DMPC, the zwitterionic analog which has the same hydrophobic domain of $\mathrm{C} 14$ atoms chains and is organized as a typical gel phase bilayer (Oliveira et al. 2012) (Fig. 6, at the top, shows, for comparison, the spectra of the different labels incorporated in DMPC and diC14-amidine bilayers at the gel phase). Consistently, $A_{\max }$ values of all probes embedded in diC14-amidine are much larger than the ones embedded in DMPC, and they are also less dependent on temperature (Fig. 6). The absence of a flexibility gradient and the increase in rigidity suggests that diC14-amidine organizes as an 
DMPC
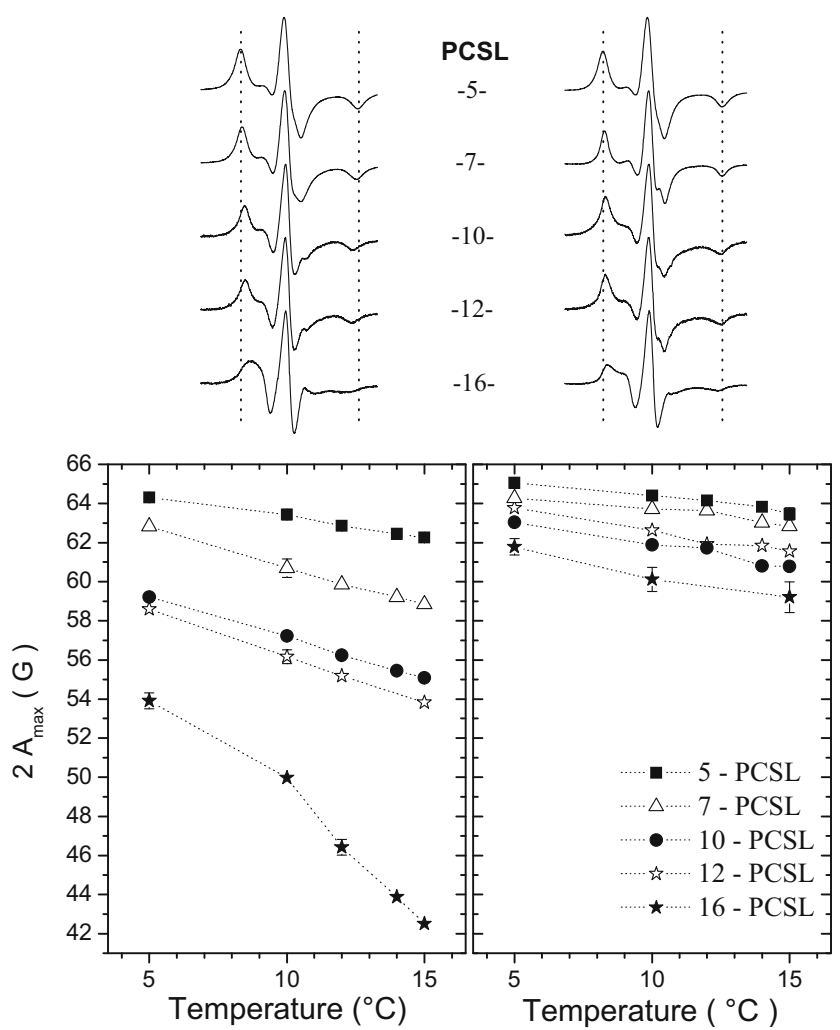

Fig. 6 Upper figures show ESR spectra of 5-, 7-, 10-, 12- and 16-PCSL incorporated in DMPC and diC14-amidine, at $5{ }^{\circ} \mathrm{C}$. Dotted lines indicate the positions of the outer features of the 5-PCSL spectra. Total spectra width $100 \mathrm{G}$. Lower graphs show the outer hyperfine splitting $\left(2 A_{\max }\right)$ measured on ESR spectra of different spin labels incorporated in the gel phase of DMPC and diC14-amidine. (Reprinted from Oliveira et al. 2012, copyright 2012 American Chemical Society)

interdigitated gel phase (Oliveira et al. 2012). In fact, the absence of a flexibility gradient in the gel phase detected by ESR spectroscopy has been employed as a fast and straightforward method to diagnose interdigitation: in this case, ESR probes located near the bilayer core have less freedom of motion and are more ordered than those in non-interdigitated bilayers, and they have empirical parameters similar to those of the probes near the bilayer surface (Hirsh et al. 1998; Boggs and Rangaraj 1985; Boggs et al. 1989).

Interdigitation results in very ordered phases where the two bilayer leaflets are merged; for symmetric lipids, it is usually a consequence of interfacial perturbations, such as modifications of the polar headgroup area or dehydration of the bilayer (Wu et al. 2010). Interdigitation has been described for cationic lipids presenting large headgroups and short acyl chains or high surface charge density (Pohle et al. 2000; Lewis et al. 2001; Winter et al. 2001; Ryhanen et al. 2005). In those cases, the electrostatic repulsion between headgroups played a major role, since addition of neutral lipids or salt affected interdigitation (Ryhanen et al. 2005).
Interestingly, DMPG, a hydrocarbon chain analog of diC14-amidine with a large and charged anionic headgroup, does not form interdigitated gel phase bilayers (Pabst et al. 2007, 2012). This difference could be explained by the lipid backbones: while in DMPG the myristic chains are separated by four atoms, in diC14-amidine these chains are separated by five atoms. Apparently, interdigitation of the diC14amidine gel phase bilayers does not depend exclusively on the interactions between charged headgroups, but also on the architecture of the lipid backbone. Consistently, interdigitation due to increased intramolecular chain separation is also observed in gel phase bilayers of 1,3-DPPC, a synthetic isomer of zwitterionic DPPC (Serrallach et al. 1983; Zumbuehl et al. 2014).

Moreover, increasing the size of hydrocarbon chains did not prevent interdigitation in gel phase bilayers of diC16amidine, a 16-carbon lipid chain (Fig. 1) derivative of diC14-amidine (Rozenfeld et al. 2015b). Bilayers of diC16amidine and DPPC, which have the same hydrophobic domain, were analyzed from surface to core using 5-, 7-, 10-, 12-, 14- and 16-PCSL spin labels. While DPPC presents the expected flexibility gradient toward the core, in diC16amidine bilayers this gradient exists only down to the 12th carbon. The 14th and 16th carbons of diC16-amidine are in a very rigid environment, as shown by the more anisotropic spectra and higher $A_{\max }$ values for these probes (Rozenfeld et al. 2015b). This suggests that diC16-amidine molecules are organized in a partially interdigitated gel phase.

Interdigitation has been implied in the fusion process of phospholipid membranes induced by ethanol (Ahl et al. 1994; Komatsu and Okada 1995; Ahl and Perkins 2003; Polozova et al. 2005). Accordingly, diC14- and diC16amidine are highly fusogenic at those temperatures at which interdigitated gel phases were observed (Oliveira et al. 2012; Rozenfeld et al. 2015b). The results of X-ray and neutron scattering experiments suggest that interdigitated diC14amidine molecules were packed side by side and flipped horizontally from one to the other (Pabst et al. 2012). This adjacent interdigitated organization leads to a significant exposure of the hydrophobic liquid core to the aqueous solvent, but reduces the energy penalties associated with water removal during the fusion process and could explain the high fusogenic efficiency of diC14-amidine in the gel phase (Pabst et al. 2012).

In the fluid phase, diC14- and diC16-amidine adopt a lamellar bilayer organization, with a typical flexibility gradient observed from the decrease in ESR spectra anisotropy toward the bilayer core (Oliveira et al. 2012; Rozenfeld et al. 2015b). Empirical parameters, suitable to be used in the bilayer fluid phase, calculated from spectra features, such as the $h_{+1} / h_{0}$ ratio, the effective order parameter $\left(S_{\text {eff }}\right)$ and rotational correlation times $\tau_{B}$ and $\tau_{C}$, show that the fluid phases of the diC14- and diC16-amidine are more rigid than the ones from 
their zwitterionic analogs, DMPC and DPPC, respectively, both close to the bilayer surface, monitored by 5 - and 7 PCSL, and at the bilayer core, monitored by 16-PCSL (Oliveira et al. 2012; Rozenfeld et al. 2015b). This is an interesting result that still needs to be further understood, but it is certainly related to the dimensions and interactions of the lipid headgroups. It is worth mentioning that these empirical parameters also show that the fluid phase of diC16-amidine is more packed than that of diC14-amidine, as expected from the enhanced hydrophobic interactions resulting from longer hydrocarbon chains (Oliveira et al. 2012; Rozenfeld et al. 2015b).

At $\mathrm{pH}$ 3.0, where diC14-amidine is double-charged, ESR spectra and empirical parameters (such as $A_{\max }$ and $h_{+} / h_{0}$ ratio) show that the gel phase is less tightly packed and organized than at pH 7.4 (Fig. 3a, b). Furthermore, at pH 3.0, it is possible to observe, on the ESR spectra, that the membrane core is more fluid than the surface, implying that there is a flexibility gradient in the gel phase membrane, i.e., at $\mathrm{pH} 3.0$ the gel phase of diC14-amidine is not interdigitated.

\section{Detecting DODAB bicelles by ESR}

The cylindrical geometry of DODAB molecules (Fig. 1) allows the formation of different structural ensembles in aqueous solutions: large vesicles are produced by chloroform vaporization (Carmona-Ribeiro and Chaimovich 1983) or heating above the phase transition temperature (Tsuruta et al. 1997); small open bilayer fragments (or bicelles) are produced by sonication (Pansu et al. 1990; Andersson et al. 1995; Benatti et al. 2001). Vesicle sizes were found to be highly polydisperse. While the vesicles showed a mean hydrodynamic diameter of around $400 \mathrm{~nm}$ (Carmona-Ribeiro and Chaimovich 1983), giant vesicles with a diameter of up to $10 \mu \mathrm{m}$ were observed by optical microscopy (Oliveira et al. 2011) (Fig. 7). The sonicated bilayer fragments are also polydisperse, but have mean hydrodynamic sizes around $90 \mathrm{~nm}$ (Vieira et al. 2006; Rozenfeld et al. 2011).

Spin labels embedded in sonicated DODAB dispersions showed the coexistence of gel and fluid domains at temperatures far below the phase transition temperature (Benatti et al. 2001). This coexistence of phases at low temperatures was absent in DODAB large vesicles and was interpreted as an indication of the presence of highly curved fluid edges in sonicated samples, which would be expected in bicelles (See sketch in Fig. 8a).

In fact, it was possible to estimate the fractions of gel and fluid domains from spectra simulations, as discussed above, since the simulated spectra could be split into two ESR signals, corresponding to spin labels in different domains, namely, a fluid and a gel domain (Oliveira et al. 2011) (Fig. 9).

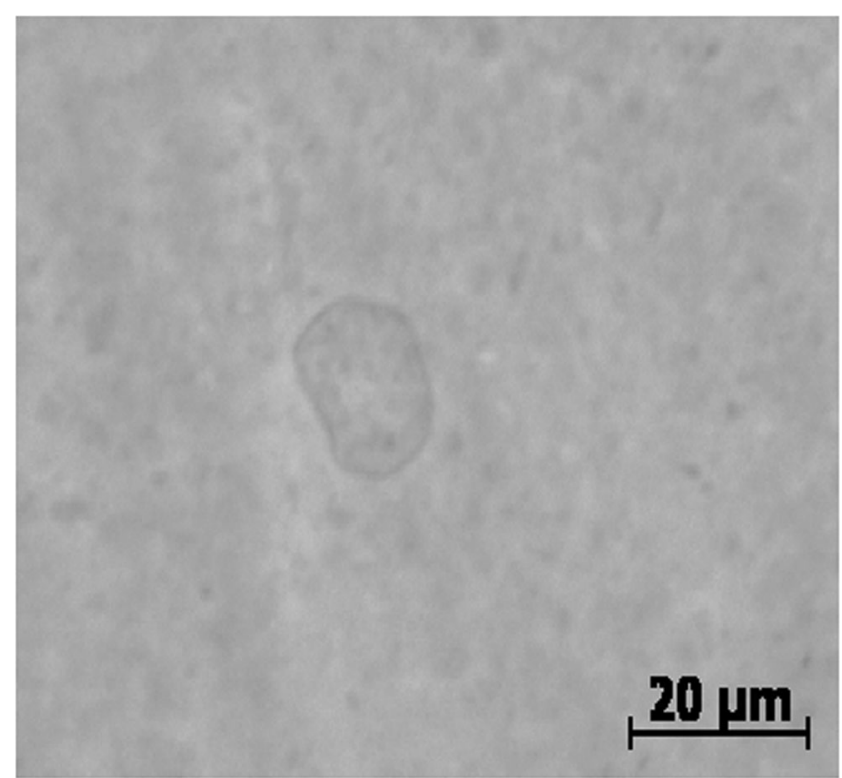

Fig. 7 A typical optical microscopy image of non-sonicated DODAB dispersion prepared by hydrating crystals of the amphiphile in water at $57^{\circ} \mathrm{C}$ for $20 \mathrm{~min}$ to the final concentration of $2 \mathrm{mM}$. Dispersions were vortexed for $5 \mathrm{~min}$ and kept at room temperature for $3 \mathrm{~h}$ to stabilize. (Experiment conduced in collaboration with KA Riske)

The coexistence of phases at low temperatures was later confirmed by ${ }^{1} \mathrm{H}$-nuclear magnetic resonance (Cocquyt et al. 2004) and is consistent with DSC data that show a large decrease in transition enthalpy for sonicated membranes, thereby suggesting the absence of large regions of DODAB in the gel phase (Cocquyt et al. 2004; Rozenfeld et al. 2011). These fluid
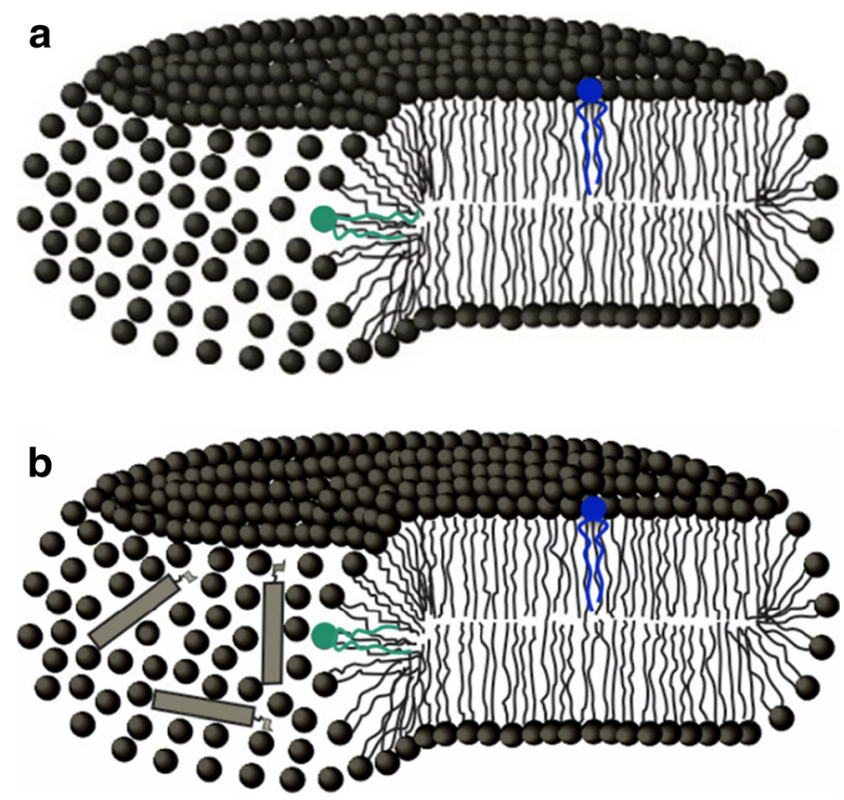

Fig. 8 Schematic representation of a DODAB bicelle (a) and amphotericin $\mathrm{B}(\mathrm{AmB})$ bound to a DODAB bicelle $(\mathbf{b}) . \mathrm{AmB}$ is represented as a rigid rectangle with the sugar moiety. The colored lipids represent spin labels in the two different domains: the gel bilayer (blue) and the fluid borders (green). (Adapted from Oliveira et al. 2011, copyright 2011 Elsevier) 


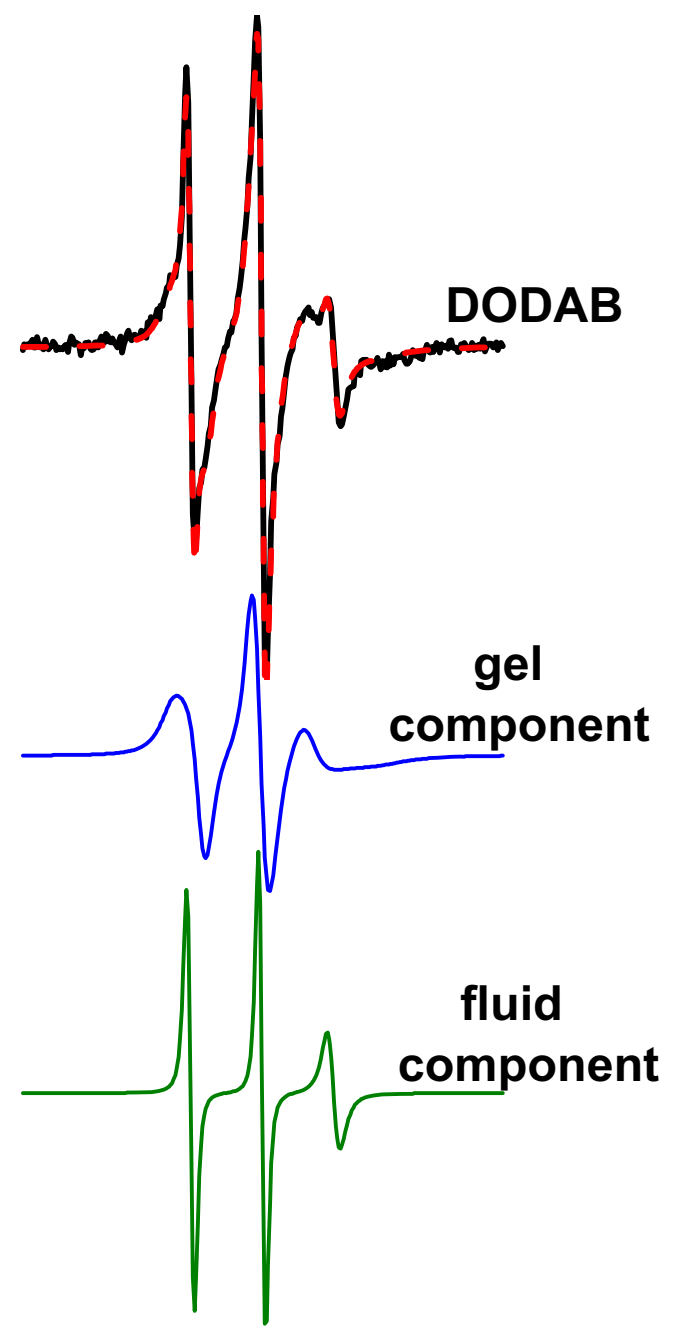

Fig. 9 Electron spin resonance spectra of 16-MESL incorporated in DODAB. Top ESR spectra: solid lines correspond to the experimental spectra of DODAB, red dashed line corresponds to the best fit simulation. Middle and bottom ESR spectra: the two components obtained from the spectral simulation, $T=30{ }^{\circ} \mathrm{C}$. (adapted from Oliveira et al. 2011, Copyright 2011 Elsevier)

phases at the edges of bicelles would favor the adsorption of hydrophobic molecules, as discussed in the following sections.

\section{Characterization of the interaction between cationic membranes and other molecules of biological interest}

\section{Amphotericin B}

Amphotericin B (AmB) (Fig. 1) is a polyene antifungal drug widely used for the treatment of systemic fungal infections (Hartsel and Bolard 1996; Denning and Hope 2010; Gallis et al. 1990). Its mechanism of action is supposed to be based on changes in membrane permeability (Bolard 1986; Fujii et al. 1997; Cohen 2010), although a mechanism based on the production of free radicals has also been suggested (Lamy-Freund et al. 1985; Sokol-Anderson et al. 1988; Sangalli-Leite et al. 2011). The use of AmB is limited by several side effects, including nephrotoxicity and hemolytic activity; therefore much effort has been made to develop formulations able to reduce its toxicity and increase its therapeutic effect (Brajtburg and Bolard 1996; Torrado et al. 2008). Both the therapeutic and toxic effects of AmB seem to be related to its state of aggregation, and the monomeric state of AmB is usually ascribed as the less toxic state (LamyFreund et al. 1989; Bolard et al. 1991; Barwicz and Tancrede 1997; Barwicz et al. 1992; Legrand et al. 1992). In this context, sonicated DODAB bicelles have been found to be able to solubilize mainly monomeric $\mathrm{AmB}$ molecules (Vieira and Carmona-Ribeiro 2001) and display low nephrotoxicity (Lincopan et al. 2005).

Since spin labels can detect gel and fluid domains, simulations of ESR spectra have allowed the independent analysis of the effect of AmB on the bulk and edges of DODAB bicelles (Oliveira et al. 2011). The fractions of the gel and fluid domains were not altered by the presence of $\mathrm{AmB}$, suggesting that no major rearrangement of the bilayer (such as fusion) took place. Most spectral changes were observed in the shape of the fluid domain component: AmB rigidifies the fluid domains, resulting in increases of rotational correlation time and order parameter of the spin labels and decreases of the bilayer polarity (Fig. 10). The larger effect on the fluid component of the spectra indicated that $\mathrm{AmB}$ is mostly bound at the edges of the DODAB bicelles (Fig. 8b). For temperatures above the gel-fluid transition, AmB slightly increased the bilayer fluidity, which was already fluid (Oliveira et al. 2011). The preferential binding of $\mathrm{AmB}$ to the highly disorganized edges of DODAB bicelles suggests that the same strategy could be employed for other formulations, such as mixtures of natural lipids known to form bicelles (Katsaras et al. 2005).

\section{Cholesterol}

Cholesterol is an abundant component of plasma membranes, being essential for mammalian cell viability and proliferation (Kulig et al. 2016; Howe et al. 2016). The structure of cholesterol molecules, with a flat and stiff steroid moiety linked to a hydroxyl group (Fig. 1), affects membrane packing, elasticity and accessibility to small water-soluble molecules (Kulig et al. 2016; Howe et al. 2016). Hence, cholesterol is involved in several biological processes by altering the properties of lipid bilayers: it participates in lipid nanodomain formation (Edidin 2003; Simons and Sampaio 2011), regulates protein functions (Epand 2006; Kuwabara and Labouesse 2002) and is a precursor of steroid hormones and bile acids (Chiang 2004). On the other hand, dysregulation of cholesterol homeostasis is involved with pathologies such as atherosclerosis, diabetes, 


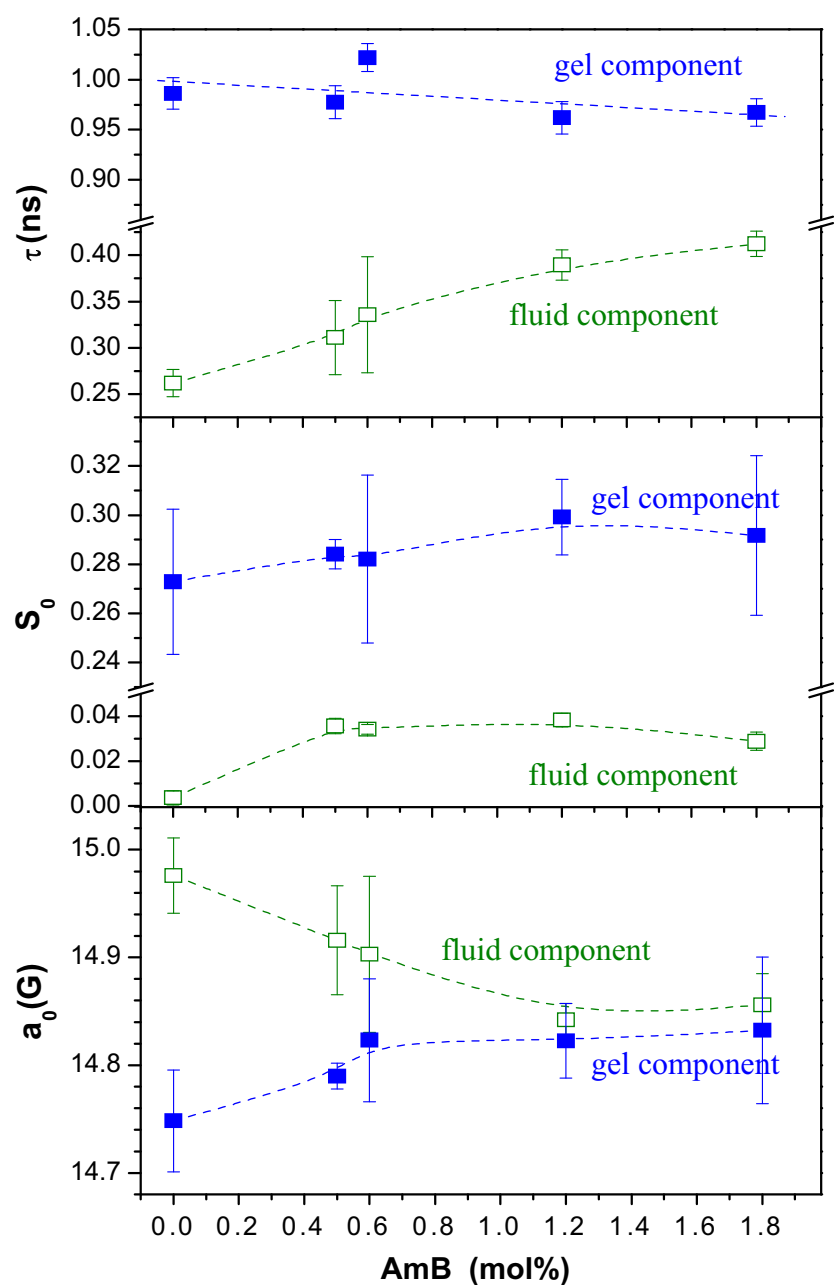

Fig. 10 Best simulated values of the rotational correlation time $(\tau)$, order parameter $\left(S_{o}\right)$ and isotropic hyperfine splitting $\left(a_{o}\right)$ as a function of the AmB-DODAB molar ratio, for the fluid component (open symbols) and the gel component (closed symbols) of 16-MESL in DODAB aggregates. (Adapted from Oliveira et al. 2011, copyright 2011 Elsevier)

cancer and dementia (Kulig et al. 2016; Lange and Steck 2016).

ESR spectra of 5- and 16-PCSL spin labels show that cholesterol has low solubility in DODAB bilayers since the coexistence of phases corresponding to DODAB and cholesterolrich domains could be detected for a cholesterol concentrations as low as $15 \mathrm{~mol} \%$ (Benatti et al. 2007) (Fig. 11). Cholesterol-rich domains could be either inside or outside the bilayer. This contrasts with the high solubility of cholesterol in phosphatidylcholine bilayers (Huang et al. 1999), but is in agreement with the poor mixing of cholesterol in anionic charged bilayers (Bach and Wachtel 2003) and in membranes composed of polyunsaturated acyl chains (Brzustowicz et al. 2002; Marquardt et al. 2016). While the latter effect can be understood from the irregular contour of the polyunsaturated chains that do not pack well with the flat surface of cholester$\mathrm{ol}$, the first can be a consequence of the contrast between the

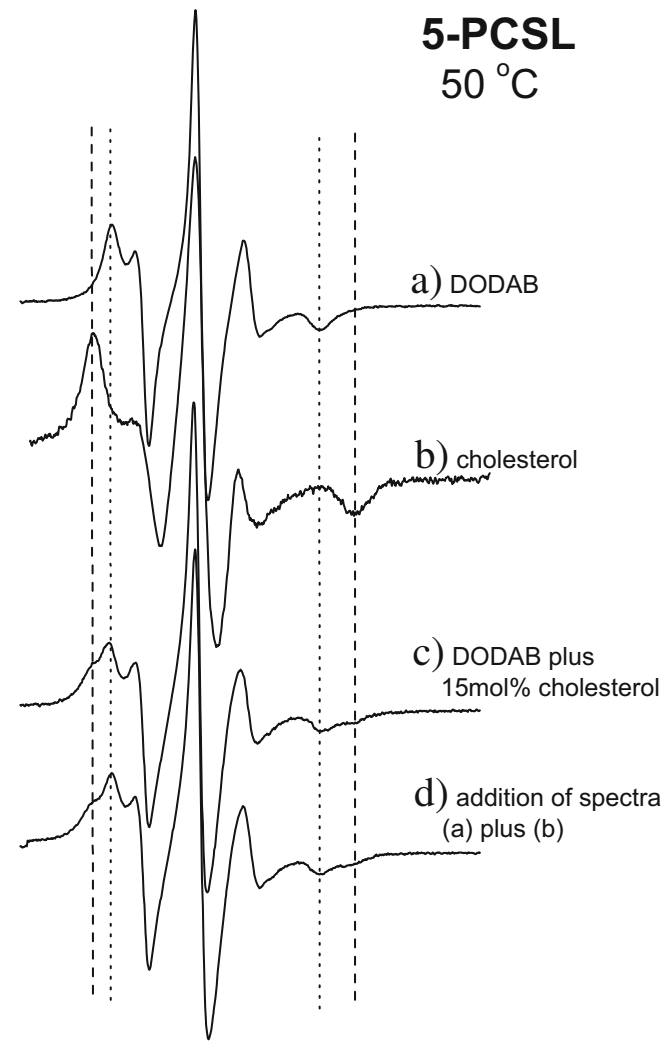

Fig. 11 a, b Electron spin resonance spectra of 5-PCSL incorporated in the DODAB bilayer (a) and in cholesterol crystals (b), at $50{ }^{\circ} \mathrm{C}$. c ESR spectrum yielded by 5-PCSL in DODAB dispersion with $15 \mathrm{~mol} \%$ of cholesterol (c). d ESM spectrum created by the addition of fractions of signals (a) and (b). (Adapted from Benatti et al. 2007, copyright 2007 Elsevier)

more hydrophilic charged group and the hydrophobic nature of cholesterol (Benatti et al. 2008).

It was also observed that cholesterol fractions below $15 \mathrm{~mol} \%$ fluidize and/or induce disorder in the gel phase while rigidifying the fluid phase of DODAB (Benatti et al. 2007). This effect is similar to the one observed in phospholipid membranes (Finean 1990; Bhattacharya and Haldar 2000).

Considering that cholesterol crystals serve as proinflammatory danger signals (Fessler 2016), the low solubility of cholesterol in DODAB bilayers should be taken into account when planning biological applications with these cationic membranes. Furthermore, the known pathophysiological effects of cholesterol derivatives (Fessler 2016; Kulig et al. 2016) suggest that testing their effects on the structure of DODAB bilayers might be interesting.

\section{DNA}

DNA has been extensively employed as a biotechnological tool, with applications in different forms of genetic modification, such as gene therapy and silencing (Fichou and Férec 2006; Hille and Charpentier 2016; Robinson-Hamm and 
DODAB

(0.2 mM labeled (2 mol\% 16-PCSL) + $1.8 \mathrm{mM}$ unlabeled) $+0.4 \mathrm{mM}$ ODN

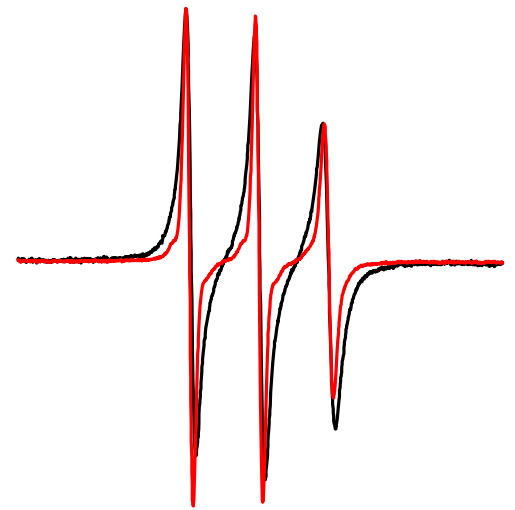

Fig. 12 Effect of the oligonucleotide on ESR spectrum of mixtures containing spin-labeled and unlabeled DODAB vesicles. Vesicles were labeled with 2 mol\% 16-PCSL. A final $0.2 \mathrm{mM}$ labeled $/ 1.8 \mathrm{mM}$ unlabeled vesicle ratio interacted in the presence or absence of the oligonucleotide for $1 \mathrm{~h}$ at room temperature before the ESR spectra were recorded at $60{ }^{\circ} \mathrm{C}$. (Adapted from Rozenfeld et al. 2015a, with permission of the PCCP Owner Societies)

Gersbach 2016), in vaccine development (Aps et al. 2016) and as vaccine adjuvants (Rozenfeld et al. 2012), and even in the delivery of macromolecules (as hydrogels and DNA cages) (Campolongo et al. 2010; Walsh et al. 2011). In many of these applications, the DNA molecules used are either in the form of long and circular plasmids (see, for example, Aps et al. 2016; Robinson-Hamm and Gersbach 2016) or as small linear oligonucleotides (Fichou and Férec 2006; Rozenfeld et al. 2012).

The effect of small single-stranded DNA oligonucleotides on the structure of DODAB vesicles has been studied with ESR spectroscopy (Rozenfeld et al. 2015a). Spin labels were used to probe oligonucleotide-induced bilayer fusion by monitoring spin-spin exchange: vesicles marked with excess spin labels display a broad ESR spectrum that is characteristic of spin exchange interactions (Devaux and McConnell 1972). Hence, vesicles labeled with excess ( $2 \mathrm{~mol} \%$ ) 16-PCSL were mixed with unlabeled vesicles at a 1:9 $\mathrm{M}$ ratio, and no spontaneous vesicle fusion was observed because the broad ESR remained unaltered. However, the addition of oligonucleotides to the vesicle mixture resulted in a narrowing of the spectrum due to spin label dilution within the bilayer phase caused by fusion (Rozenfeld et al. 2015a) (Fig. 12). Therefore, ESR spectroscopy can be used as a tool to assess bilayer fusion.

ESR spectroscopy also showed that oligonucleotides stabilize the DODAB gel phase by making it more rigid at the surface and at the core. Consistently, the coexistence of phases observed at the gel-fluid transition (Benatti et al. 2001) was shifted to higher temperatures. In the fluid phase, oligonucleotides simultaneously induced a decrease in superficial ordering and an increase in bilayer core packing (Rozenfeld et al. 2015a). It is noteworthy that the effect of oligonucleotides and monovalent salt were different: the stabilization of the gel phase by monovalent salt was only noted in the bilayer core, and the salt slightly decreased bilayer packing at both the surface and the core of DODAB fluid phases (Benatti et al. 2007). These differences suggest that dehydration effects caused by superficial adsorption of oligonucleotides could be playing a more important role in DODAB bilayer organization than electrostatic effects caused by salt (Rozenfeld et al. 2015a).

The structure of diC14-amidine was also affected by oligonucleotide adsorption: spin labels at different bilayer depths showed that electrostatic interactions between negatively charged oligonucleotides and cationic diC14-amidine
Fig. 13 Scheme of adjacent (a) and interpenetrated (b) interdigitated gel phases of diC14-amidine bilayers. (Adapted from Rozenfeld et al. 2013, copyright 2013 American Chemical Society) a)

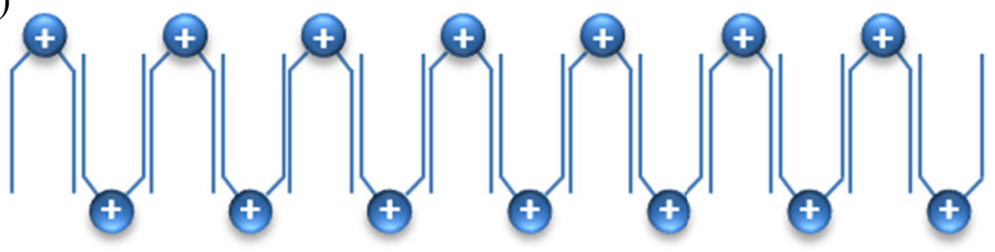

b)

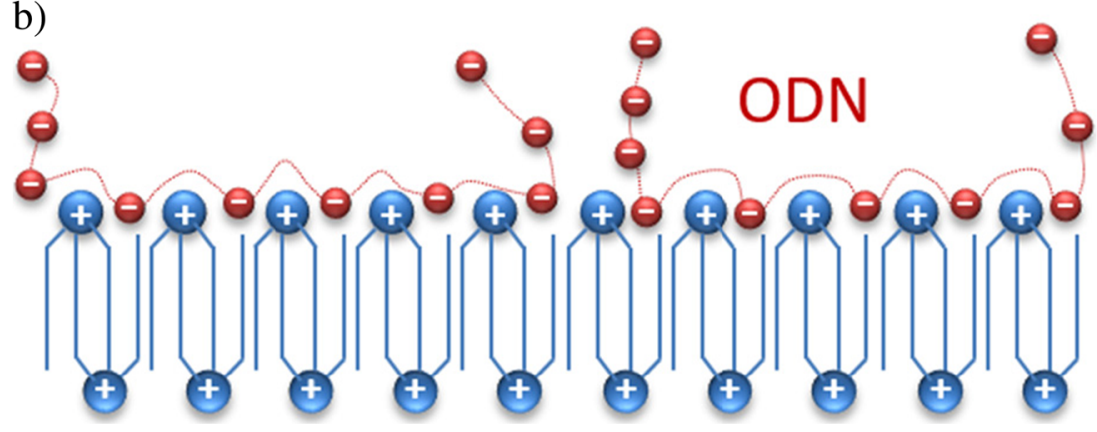


headgroups increased the rigidity of both the gel and fluid phases (Rozenfeld et al. 2013). As previously described, gel phase diC14-amidine bilayers display an interdigitated gel phase in which molecules are packed side by side and flipped horizontally from one to the other (Oliveira et al. 2012; Pabst et al. 2012). Consequently, the increase in gel phase rigidity, which did not display a flexibility gradient even in the presence of oligonucleotide, was probably due to the transformation of an adjacent to an interpenetrated interdigitation (Fig. 13).

In agreement with the results obtained with oligonucleotides, 16-PCSL spectra showed that the adsorption of longer double-stranded DNA from salmon testes also had a rigidifying effect on the gel and fluid diC14-amidine bilayers (Benatti et al. 2009). Since the structure of the cationic lipid-DNA assemblies affect their interactions with cells (Allen and Cullis 2013; ur Rehman et al. 2013; Dan and Danino 2014), the structural effects described for DODAB and diC14amidine should be taken in account when planning biotechnological applications, such as delivery systems.

\section{Final remarks}

In this review we have demonstrated how ESR spectroscopy has provided important structural information on aggregates formed by the cationic lipids DODAB and diC14-amidine. ESR spectroscopy has made a relevant contribution to our current understanding of bilayer thermotropic behavior, gel and fluid bilayer phase structures, coexistence of bilayer phases, presence of bilayer interdigitation, membrane fusion and structural modifications due to the interaction of the cationic membranes with other biologically relevant molecules.

Acknowledgements This work was supported by FAPESP, CNPq, CAPES, NAP-FCx (USP). Additionally, JHKR acknowledges grant \#2016/19077-1 FAPESP, and MTL acknowledges research fellowship from CNPq.

This article does not contain any studies with human or animal subjects performed by any of the authors.

\section{Compliance with ethical standards}

Conflict of interest All authors declare that they have no conflicts of interest.

Ethical approval This article does not contain any studies with human participants or animals performed by any of the authors.

\section{References}

Ahl PL, Perkins WR (2003) Interdigitation-fusion liposomes. Methods Enzymol 367:80-98

Ahl PL, Chen L, Perkins WR, Minchey SR, Boni LT, Taraschi TF, Janoff AS (1994) Interdigitation-fusion: a new method for producing lipid vesicles of high internal volume. Biochim Biophys Acta 1195(2): 237-244

Allen TM, Cullis PR (2013) Liposomal drug delivery systems: from concept to clinical applications. Adv Drug Deliv Rev 65(1):36-48

Althenbach C (2017) The program is written in LabVIEW (National Instruments) and can be freely downloaded from the following site: http://www.chemistry.ucla.edu/directory/hubbell-wayne-1

Andersson M, Hammarström L, Edwards K (1995) Effect of bilayer phase transitions on vesicle structure and its influence on the kinetics of viologen reduction. J Phys Chem 99:14531-14538

Anyarambhatla GR, Needham D (1999) Enhancement of the phase transition permeability of DPPC Liposomes by incorporation of MPPC: a new temperature-sensitive liposome for use with mild hyperthermia. J Liposome Res 9(4):491-506

Aps LRMM, Tavares MB, Rozenfeld JHK, Lamy MT, Ferreira LCS, Diniz MO (2016) Bacterial spores as particulate carriers for gene gun delivery of plasmid DNA. J Biotechnol 228:58-66

Bach D, Wachtel E (2003) Phospholipid/cholesterol model membranes: formation of cholesterol crystallites. Biochim Biophys Acta 1610(2):187-197

Barwicz J, Tancrede P (1997) The effect of aggregation state of amphotericin-B on its interactions with cholesterol- or ergosterolcontaining phosphatidylcholine monolayers. Chem Phys Lipids 85(2):145-155

Barwicz J, Christian S, Gruda I (1992) Effects of aggregation state of amphotericin B on its toxicity to mice. Antimicrob Agents Chemother 36(10):2310-2315

Benatti CR, Tiera MJ, Feitosa E, Olofsson G (1999) Phase behavior of synthetic amphiphile vesicles investigated by calorimetry and fluorescence methods. Thermochim Acta 328(1-2):137-142

Benatti CR, Feitosa E, Fernandez RM, Lamy-Freund MT (2001) Structural and thermal characterization of dioctadecyldimethylammonium bromide dispersions by spin labels. Chem Phys Lipids 111(2):93-104

Benatti CR, Ruysschaert JM, Lamy MT (2004) Structural characterization of diC14-amidine, a pH-sensitive cationic lipid used for transfection. Chem Phys Lipids 131(2):197-204

Benatti CR, Epand RM, Lamy MT (2007) Low cholesterol solubility in DODAB liposomes. Chem Phys Lipids 145(1):27-36

Benatti CR, Lamy MT, Epand RM (2008) Cationic amphiphiles and the solubilization of cholesterol crystallites in membrane bilayers. Biochim Biophys Acta 1778(4):844-853

Benatti CR, Barroso RP, Lonez C, Ruysschaert JM, Lamy MT (2009) DNA alters the bilayer structure of cationic lipid diC14-amidine: a spin label study. Biochim Biophys Acta 1788(6):1304-1309

Bertrand N, Leroux JC (2012) The journey of a drug-carrier in the body: an anatomo-physiological perspective. J Control Release 161(2): $152-163$

Bhattacharya S, Haldar S (2000) Interactions between cholesterol and lipids in bilayer membranes. Role of lipid headgroup and hydrocarbon chain-backbone linkage. Biochim Biophys Acta 1467(1):39-53

Blandamer MJ, Briggs B, Cullis PM, Green JA, Waters M, Soldi G (1992) Differential scanning microcalorimetric study of vesicles in aqueous solutions formed by dimethyldioctadecylammonium bromide. J Chem Soc Faraday Trans 88(23):3431-3434

Blandamer MJ, Briggs B, Cullis PM, Engberts JBFN, Norman RI (1996) Influence of polar headgroups on the gel to liquid-crystal transition in vesicular and lipid bilayer systems. J Chem Soc Faraday Trans 92(17):3163-3164

Boggs JM, Rangaraj G (1985) Phase transitions and fatty acid spin label behavior in interdigitated lipid phases induced by glycerol and polymyxin. Biochim Biophys Acta 816(2):221-233

Boggs JM, Rangaraj G, Watts A (1989) Behavior of spin labels in a variety of interdigitated lipid bilayers. Biochim Biophys Acta 981(2):243-253 
Bolard J (1986) How do the polyene macrolide antibiotics affect the cellular membrane-properties. Biochim Biophys Acta 864(3-4): 257-304

Bolard J, Legrand P, Heitz F, Cybulska B (1991) One-sided action of amphotericin $\mathrm{B}$ on cholesterol-containing membranes is determined by its self-association in the medium. Biochemistry 30(23):57075715

Brajtburg J, Bolard J (1996) Carrier effects on biological activity of amphotericin B. Clin Microbial Rev 9(4):512-531

Brzustowicz MR, Cherezov V, Zerouga M, Caffrey M, Stillwell W, Wassall SR (2002) Controlling membrane cholesterol content. A role for polyunsaturated (docosahexaenoate) phospholipids. Biochemistry 41(41):12509-12519

Budil DE, Lee S, Saxena S, Freed JH (1996) Nonlinear-least-squares analysis of slow-motion EPR spectra in one and two dimensions using a modified Levenberg-Marquardt algorithm. J Magn Reson 120(2):155-189

Campolongo MJ, Tan SJ, Xu J, Luo D (2010) DNA nanomedicine: engineering DNA as a polymer for therapeutic and diagnostic applications. Adv Drug Deliv Rev 62(6):606-616

Caracciolo G, Amenitsch H (2012) Cationic liposome/DNA complexes: from structure to interactions with cellular membranes. Eur Biophys J 41(10):815-829

Carmona-Ribeiro AM, Chaimovich H (1983) Preparation and characterization of large dioctadecyldimethylammonium chloride liposomes and comparison with small sonicated vesicles. Biochim Biophys Acta 733(1):172-179

Chiang JYL (2004) Regulation of bile acid synthesis: pathways, nuclear receptors, and mechanisms. J Hepatol 40(3):539-551

Cocquyt J, Olsson U, Olofsson G, Van der Meeren P (2004) Temperature quenched DODAB dispersions: fluid and solid state coexistence and complex formation with oppositely charged surfactant. Langmuir 20(10):3906-3912

Cocquyt J, Olsson U, Olofsson G, Van der Meeren P (2005) Thermal transitions of DODAB vesicular dispersions. Colloid Polym Sci 283:1376-1381

Cohen BE (2010) Amphotericin B membrane action: role for two types of ion channels in eliciting cell survival and lethal effects. J Membr Biol 238:1-20

Dan N, Danino D (2014) Structure and kinetics of lipid-nucleic acid complexes. Adv Colloid Interf Sci 205:230-239

Denning DW, Hope WW (2010) Therapy for fungal diseases: opportunities and priorities. Trends Microbiol 18(5):195-204

Devaux P, McConnell HM (1972) Lateral diffusion in spin-labeled phosphatidylcholine multilayers. J Am Chem Soc 94(13):4475-4481

Edidin M (2003) The state of lipid rafts: from model membranes to cells. Annu Rev Biophys Biomol Struct 32:257-283

El Ouahabi A, Pector V, Fuks R, Vandenbranden M, Ruysschaert JM (1996) Double long-chain amidine liposome-mediated self replicating RNA transfection. FEBS Lett 380(1):108-112

Engberts JBFN, Hoekstra D (1995) Vesicle-forming synthetic amphiphiles. Biochim Biophys Acta 1241(3):323-340

Epand RM (2006) Cholesterol and the interaction of proteins with membrane domains. Prog Lipid Res 45(4):279-294

Evans D, Wennerström H (1999) The colloidal domain: where physics, chemistry, biology and technology meet. Wiley, New York

Fessler MB (2016) The intracellular cholesterol landscape: dynamic integrator of the immune response. Trends Immunol 37(12):819-830

Fichou Y, Férec C (2006) The potential of oligonucleotides for therapeutic applications. Trends Biotechnol 24(12):563-570

Finean JB (1990) Interaction between cholesterol and phospholipid in hydrated bilayers. Chem Phys Lipids 54(3-4):147-156

Freed JH (1976) Theory of slow tumbling ESR spectra for nitroxides. In: Berliner LJ (ed) Spin Labeling: theory and applications, vol 1. Academic Press, New York, pp 53-132
Fujii G, Chang JE, Coley T, Steere B (1997) The formation of amphotericin B ion channels in lipid bilayers. Biochemistry 36(16):4959-4968

Gaffney BJ (1976) Practical considerations for the calculation of order parameter for fatty acid or phospholipid spin labels in membranes. In: Berliner LJ (ed) Spin labeling: theory and applications, vol 1. Academic Press, New York, pp 567-571

Gallis HA, Drew RH, Pickard WW (1990) Amphotericin-B - 30 years of clinical experience. Rev Infect Dis 12(2):308-329

Griffith OH, Dehlinger PJ, Van SP (1974) Shape of the hydrophobic barrier of phospholipid bilayers (evidence for water penetration in biological membranes). J Membrane Biol 15(2):159-192

Hartsel S, Bolard J (1996) Amphotericin B: new life for an old drug. Trends Pharmacol 17(12):445-449

Hille F, Charpentier E (2016) CRISPR-Cas: biology, mechanisms and relevance. Phil Trans R Soc B 371(1707): 20150496. doi:10.1098/ rstb.2015.0496

Hirsh DJ, Lazaro N, Wright LR, Boggs JM, McIntosh TJ, Schaefer J, Blazyk J (1998) A new monofluorinated phosphatidylcholine forms interdigitated bilayers. Biophys J 75(4):1858-1868

Howe V, Sharpe LJ, Alexopoulos SJ, Kunze SV, Chua NK, Li D, Brown AJ (2016) Cholesterol homeostasis: how do cells sense sterol excess? Chem Phys Lipids 199:170-178

Huang J, Buboltz JT, Feigenson GW (1999) Maximum solubility of cholesterol in phosphatidylcholine and phosphatidylethanolamine bilayers. Biochim Biophys Acta 1417(1):89-100

Hubbell WL, McConnell HM (1971) Molecular motion in spin-labeled phospholipids and membranes. J Am Chem Soc 93(2):314-326

Hubert DHW, Jung M, German AL (2000) Vesicle templating. Adv Mater 12(17):1291-1294

Junquera E, Aicart E (2016) Recent progress in gene therapy to deliver nucleic acids with multivalent cationic vectors. Adv Colloid Interf Sci 233:161-175

Katsaras J, Harroun TA, Pencer J, Nieh MP (2005) "Bicellar" lipid mixtures as used in biochemical and biophysical studies. Naturwissenschaften 92(8):355-366

Knowles PF, Marsh D, Rattle HWE (1976) Magnetic resonance of biomolecules: an introduction to the theory and practice of NMR and ESR in biological systems. Wiley, London

Komatsu H, Okada S (1995) Ethanol-induced aggregation and fusion of small phosphatidylcholine liposome: participation of interdigitated membrane formation in their processes. Biochim Biophys Acta 1235(2):270-280

Kulig W, Cwiklik L, Jurkiewicz P, Rog T, Vattulainen I (2016) Cholesterol oxidation products and their biological importance. Chem Phys Lipids 199:144-160

Kuwabara PE, Labouesse M (2002) The sterol-sensing domain: multiple families, a unique role? Trends Genet 18(4):193-201

Lamy-Freund MT, Ferreira VFN, Schreier S (1985) Mechanism of inactivation of the polyene antibiotic amphotericin B - evidence for radical formation in the process of autooxidation. J Antibiot 38(6):753757

Lamy-Freund MT, Ferreira VFN, Schreier S (1989) Polydispersity of aggregates formed by the polyene antibiotic amphotericin B and deoxycholate. A spin label study. Biochim Biophys Acta 981:207212

Lange Y, Steck TL (2016) Active membrane cholesterol as a physiological effector. Chem Phys Lipids 199:74-93

Lange A, Marsh D, Wassmer KH, Meier P, Kothe G (1985) Electron spin resonance study of phospholipid membranes employing a comprehensive line-shape model. Biochemistry 24:4383-4392

Legrand P, Romero EA, Cohen BE, Bolard J (1992) Effects of aggregation and solvent on the toxicity of amphotericin B to human erythrocytes. Antimicrob Agents Chemother 36(11):2518-2522

Lewis RN, Winter I, Kriechbaum M, Lohner K, McElhaney RN (2001) Studies of the structure and organization of cationic lipid bilayer 
membranes: calorimetric, spectroscopic, and X-ray diffraction studies of linear saturated P-O-ethyl phosphatidylcholines. Biophys J 80(3):1329-1342

Lincopan N, Mamizuka EM, Carmona-Ribeiro AM (2005) Low nephrotoxicity of an effective amphotericin B formulation with cationic bilayer fragments. J Antimicrob Chemother 55(5):727-734

Majzoub RN, Ewert KK, Safinya CR (2016) Cationic liposome-nucleic acid nanoparticle assemblies with applications in gene delivery and gene silencing. Phil Trans R Soc A 374(2072):20150129

Marquardt D, Heberle FA, Greathouse DV, Koeppe RE II, Standaert RF, Van Oosten BJ, Harroun TA, Kinnun JJ, Williams JA, Wassall SR, Katsaras J (2016) Lipid bilayer thickness determines cholesterol's location in model membranes. Soft Matter 12:9417-9428

Marsh D (1981) Electron spin resonance: spin labels. In: Grell E (ed) Molecular biology biochemistry and biophysics: membrane spectroscopy. Springer, Berlin Heidelberg, New York, pp 51-142

Mason JT (1998) Investigation of phase transitions in bilayer membranes. Methods Enzymol 295:468-494

Oliveira TR, Benatti CR, Lamy MT (2011) Strucutral characterization of the interaction of the polyene antibiotic Amphotericin B with DODAB bicelles and vesicles. Biochim Biophys Acta 1808(11): 2629-2637

Oliveira TR, Duarte EL, Lamy MT, Vandenbranden M, Ruysschaert JM, Lonez C (2012) Temperature-dependence of cationic lipid bilayer intermixing: possible role of interdigitation. Langmuir 28(10):4640 4647

Pabst G, Danner S, Karmakar S, Deutsch G, Raghunathan VA (2007) On the propensity of phosphatidylglycerols to form interdigitated phases. Biophys J 93(2):513-525

Pabst G, Lonez C, Vandenbranden M, Jestin J, Radulescu A, Ruysschaert JM, Gutberlet T (2012) Stalk-free membrane fusion of cationic lipids via an interdigitated phase. Soft Matter 8:7243-7249

Pansu RB, Arrio B, Roncin J, Faure J (1990) Vesicles versus membranefragments in DODAB suspensions. J Phys Chem 94:796-801

Pector V, Caspers J, Banerjee S, Deriemaeker L, Fuks R, El Ouahabi A, Vandenbranden M, Finsy R, Ruysschaert JM (1998) Physicochemical characterization of a double long-chain cationic amphiphile (Vectamidine) by microelectrophoresis. Biochim Biophys Acta 1372(2):339-346

Pohle W, Selle C, Gauger DR, Zantl R, Artzner F, Radler JO (2000) FTIR spectroscopic characterization of a cationic lipid-DNA complex and its components. Phys Chem Chem Phys 2:4642-4650

Polozova A, Li X, Shangguan T, Meers P, Schuette DR, Ando N, Gruner SM, Perkins WR (2005) Formation of homogeneous unilamellar liposomes from as interdigitated matrix. Biochim Biophys Acta 1668(1):117-125

Ponce AM, Vujaskovic Z, Yuan F, Needham D, Dewhirst MW (2006) Hyperthermia mediated liposomal drug delivery. Int J Hyperth 22(3):205-213

Ragioto DAMT, Carrasco LDM, Carmona-Ribeiro AMC (2014) Novel gramicidin formulations in cationic lipid as broad-spectrum microbicidal agents. Int J Nanomedicine 9(1):3183-3192

Riske KA, Fernandez RM, Nascimento OR, Bales BL, Lamy-Freund MT (2003) DMPG gel-fluid thermal transition monitored by a phospholipid spin labeled at the acyl chain end. Chem Phys Lipids 124(1): 69-80

Riske KA, Barroso RP, Vequi-Suplicy CC, Germano R, Henriques VB, Lamy MT (2009) Lipid bilayer pre-transition as the beginning of the melting process. Biochim Biophys Acta Biomembr 1788(5):954 963

Robinson-Hamm JN, Gersbach CA (2016) Gene therapies that restore dystrophin expression for the treatment of Duchenne muscular dystrophy. Hum Genet 135(9):1029-1040

Rosa H, Petri DFS, Carmona-Ribeiro AM (2008) Interactions between bacteriophage DNA and cationic biomimetic particles. J Phys Chem B 112(51):16422-16430
Rozenfeld JHK, Ribeiro TR, Lamy MT, Carmona-Ribeiro AM (2011) Interaction of cationic bilayer fragments with a model oligonucleotide. Biochim Biophys Acta Biomembr 1808(3):649-655

Rozenfeld JHK, Silva SR, Ranéia PA, Faquim-Mauro E, CarmonaRibeiro AM (2012) Stable assemblies of cationic bilayer fragments and $\mathrm{CpG}$ oligonucleotide with enhanced immunoadjuvant activity in vivo. J Control Release 160(2):367-373

Rozenfeld JHK, Duarte EL, Oliveira TR, Lonez C, Ruysschaert JM, Lamy MT (2013) Oligonucleotide adsorption affects phase transition but not interdigitation of diC14-amidine bilayers. Langmuir 29(35):11102-11108

Rozenfeld JHK, Duarte EL, Barbosa LRS, Lamy MT (2015a) The effect of an oligonucleotide on the structure of cationic DODAB vesicles. Phys Chem Chem Phys 17:7498-7506

Rozenfeld JHK, Duarte EL, Ruysschaert JM, Lonez C, Lamy MT (2015b) Structural characterization of novel cationic diC16amidine bilayers: evidence for partial interdigitation. Biochim Biophys Acta Biomembr 1848(1):127-133

Ruysschaert JM, El Ouahabi A, Willeaume V, Huez G, Fuks R, Vandenbranden M, Di Stefano P (1994) A novel cationic amphiphile for transfection of mammalian cells. Biochem Biophys Res Commun 203(3):1622-1628

Ryhanen SJ, Alakoskela JM, Kinnunen PK (2005) Increasing surface charge density induces interdigitation in vesicles of cationic amphiphile and phosphatidylcholine. Langmuir 21(13):5707-5715

Sangalli-Leite F, Scorzoni L, Mesa-Arango AC, Casas C, Herrero E, Gianinni MJSM, Rodriguez-Tudela JL, Cuenca-Estrella M, Zaragoza O (2011) Amphotericin B mediates killing in Cruptococcus neoformans through the induction of a strong oxidative burst. Microb Infect 13(5):457-467

Saveyn P, Van der Meeren P, Cocquyt J, Drakenberg T, Olofsson G, Olsson U (2007) Incomplete lipid chain freezing of sonicated vesicular dispersions of double-tailed ionic surfactants. Langmuir 23(21): 10455-10462

Schneider DJ, Freed JH (1989) Calculating slow motional magnetic resonance spectra: a user's guide. In: Berliner LJ, Reuben J (eds) Spin labeling. Theory and applications, vol. 8. Plenum Press, New York, pp 1-76

Serrallach EN, Dijkman R, de Haas GH, Shipley GG (1983) Structure and thermotropic properties of 1,3-dipalmitoyl-glycero-2phosphocholine. J Mol Biol 170(1):155-174

Silva JPN, Oliveira ACN, Casal MPPA, Gomes AC, Coutinho PJG, Coutinho OP, Oliveira MECDR (2011) DODAB:monoolein-based lipoplexes as non-viral vectors for transfection of mammalian cells. Biochim Biophys Acta 1808(10):2440-2449

Simons K, Sampaio JL (2011) Membrane organization and lipid rafts. Cold Spring Harb Perspect Biol 3:a004697

Smith ICP, Schreier-Muccillo S, Marsh D (1976) Spin labeling. In: Pryor W (ed) Free radicals in biology, vol 1. Academic press, New York, pp 149-197

Sokol-Anderson M, Sligh JE, Elberg S, Brajtburg J, Kobayashi GS, Medoff G (1988) Role of cell defense against oxidative damage in the resistance of Candida albicans to the killing effect of amphotericin B. Antimicrob Agents Chemother 32(5):702-805

Südholter EJR, de Grip WJ, Engberts JBFN (1982) Rhodopsin reconstitution in vesicles formed from simple, fully synthetic amphiphiles. J Am Chem Soc 104(4):1069-1072

Tanaka T, Legat A, Adam E, Steuve J, Gatot JS, Vandenbranden M, Ulianov L, Lonez C, Ruysschaert JM, Muraille E, Tuynder M, Goldman M, Jacquet A (2008) DiC14-amidine cationic liposomes stimulate myeloid dendritic cells through toll-like receptor 4. Eur J Immunol 38(5):1351-1357

Torrado JJ, Espada R, Ballesteros MP, Torrado-Santiago S (2008) Amphotericin B formulations and drug targeting. J Pharm Sci 97(7):2405-2425 
Tsuruta LR, Quintilio W, Costa MHB, Carmona-Ribeiro AM (1997) Interactions between cationic liposomes and antigenic protein: the physical chemistry of the immunoadjuvant action. J Lipid Res 38: 2003-2011

ur Rehman Z, Zuhorn IS, Hoekstra D (2013) How cationic lipids transfer nucleic acids into cells and across cellular membranes: recent advances. J Control Release 166(1):46-56

Vieira DB, Carmona-Ribeiro AM (2001) Synthetic bilayer fragments for solubilization of amphotericin B. J Colloid Interf Sci 244(2):427431

Vieira DB, Pacheco LF, Carmona-Ribeiro AM (2006) Assembly of a model hydrophobic drug into cationic bilayer fragments. J Colloid Interface Sci 293(1):240-247
Walsh AS, Yin HF, Erben CM, Wood MJA, Turberfield AJ (2011) DNA cage delivery to mammalian cells. ACS Nano 5(7):5427-5432

Winter I, Pabst G, Rappolt M, Lohner K (2001) Refined structure of 1,2diacyl-P-O-ethylphosphatidylcholine bilayer membranes. Chem Phys Lipids 112(2):137-150

Wu FG, Wang NN, Tao LF, Yu ZW (2010) Acetonitrile induces nonsynchronous interdigitation and dehydration of dipalmitoylphosphatidylcholine bilayers. J Phys Chem B 114(39):12685-12691

Zelphati O, Wang Y, Kitada S, Reed JC, Felgner PL, Corbeil J (2001) Intracellular delivery of proteins with a new lipid-mediated delivery system. J Biol Chem 276(37):35103-35110

Zumbuehl A, Dobner B, Brezesinski G (2014) Phase behavior of selected artificial lipids. Curr Opin Colloid Interface Sci 19(1):17-24 